\title{
Tabulation of Combustion Chemistry via Artificial Neural Networks (ANNs): Methodology and Application to LES-PDF Simulation of Sydney Flame L
}

\author{
L. L. C. Franke, A. K. Chatzopoulos ${ }^{1}$ and Stelios Rigopoulos ${ }^{2}$ \\ Department of Mechanical Engineering, Imperial College London, Exhibition Road, South Kensington, London SW7 2AZ, \\ $U K$
}

\begin{abstract}
In this work, a methodology for the tabulation of combustion mechanisms via Artificial Neural Networks (ANNs) is presented. The objective of the methodology is to train the ANN using samples generated via an abstract problem, such that they span the composition space of a family of combustion problems. The abstract problem in this case is an ensemble of laminar flamelets with an artificial pilot in mixture fraction space to emulate ignition, of varying strain rate up to well into the extinction range. The composition space thus covered anticipates the regions visited in a typical simulation of a non-premixed flame. The ANN training consists of two-stage process: clustering of the composition space into subdomains using the SelfOrganising Map (SOM) and regression within each subdomain via the multilayer Perceptron (MLP). The approach is then employed to tabulate a mechanism of $\mathrm{CH}_{4}$-air combustion, based on GRI 1.2 and reduced via Rate-Controlled Constrained Equilibrium (RCCE) and Computational Singular Perturbation (CSP). The mechanism is then applied to simulate the Sydney Flame L, a turbulent non-premixed flame that features significant levels of local extinction and re-ignition. The flow field is resolved through Large Eddy Simulation (LES), while the transported Probability Density Function (PDF) approach is employed for modelling the turbulence-chemistry interaction and solved numerically via the Stochastic Fields method. Results demonstrate reasonable agreement with experiments, indicating that the SOM-MLP approach provides a good representation of the composition space, while the great savings in CPU time allow for a simulation to be performed with a comprehensive combustion model, such as the LES-PDF, with modest CPU resources such as a workstation.
\end{abstract}

Keywords: Mechanism reduction, mechanism tabulation, RCCE, Artificial Neural Network (ANN), PDF methods

\section{Introduction}

The simulation of turbulent combustion is essential for the effective design of combustion equipment such as IC engines and gas turbines, to attain improved energy efficiency and reduction in emissions of pollutants.

\footnotetext{
${ }^{1}$ Current address: McLaren Racing, McLaren Technology Centre, Woking, UK

${ }^{2}$ Corresponding author, email: s.rigopoulos@imperial.ac.uk
} 
The coupling of Computational Fluid Dynamics (CFD) and combustion modelling is very computationally demanding, however, due to the need for coupling the non-linear equations of chemical kinetics with fluid dynamics and transport phenomena. The bottleneck of the whole approach is the computation of chemical kinetics, which requires the numerical integration of a stiff system of ordinary differential equations (ODEs). Reaction mechanisms for simple fuels, such as methane and propane, typically involve 50-100 species and hundreds of reactions, which must be integrated over a time period at every spatial point of a grid comprising millions of cells. The problem is aggravated in the case of commercial fuels, such as kerosene, where the mechanism may even involve hundreds of chemical species and thousands of reactions, and when LES or DNS methods are employed.

Reduced mechanisms can help in bringing down the computational time required for direct integration of combustion mechanisms. In recent years, systematic methods for deriving reduced mechanisms have been developed; these include, among others, Computational Singular Perturbation (CSP) [1], Intrinsic Low Dimensional Manifolds (ILDM [2], Level of Importance (LOI) [3], Computer Assisted Reduction Mechanism Code (CARM) [4, 5], Rate-Controlled Constrained Equilibrium (RCCE) [6, 7, 8, 9] and Directed Relation Graph (DRG) [10]. Reviews of this field can be found in [11, 10, 12, 13]. Even when employing reduced mechanisms, however, the real-time integration of chemical kinetics within comprehensive methods such as LES-PDF and DNS is not feasible, and the time spent on the chemistry computations takes up the vast proportion of the total CPU time.

Further reduction in CPU time can only be obtained by tabulation approaches. The general principal of the latter is to perform a set of chemistry computations in advance and subsequently interpolate within the composition space covered in that set. The earliest example of tabulation is the Look-Up Table (LUT) [14], which is a straightforward interpolation within a uniform grid in composition space. This approach is feasible only when the number of species is very small, and has been used for simplified mechanisms comprising typically $\leq 4$ species. For large mechanisms, it is clearly not feasible to cover the entire composition space in advance, as the number of composition states on a discretised grid grows exponentially with dimensionality.

A pivotal approach towards circumventing this problem was In-Situ Adaptive Tabulation (ISAT) [15, 16], which attempts to tabulate on the fly only the parts of composition space actually visited during a simulation. Retrieval of the states is then performed via a binary tree method. While this approach does reduce considerably the computational time, it still means that many states must be calculated in real time, while the storage requirements and retrieval time also grow. The speed-up obtained can be larger when the simulation revisits the same parts of the composition space for a significant amount of time.

A different approach to tabulation can be sought by employing Artificial Neural Networks (ANNs). These are a class of machine learning methods that can be employed to perform various tasks, including nonlinear regression and function approximation. Therefore they can be employed to approximate the function implicitly defined by the integration of the chemical reaction step. Compared with the straightforward Look-up Table interpolation, ANNs have the advantage that they employ non-linear basis functions and therefore are able to approximate far more complex non-linear behaviour. They are also very fast and have very small requirements in terms of storage space and retrieval time, as all that needs to be stored is the coefficients of the approximation model. 
ANNs were first employed for tabulation of combustion chemistry by Christo et al. [17]. In this work, a 3-step reduced reaction scheme was employed to simulate a turbulent $\mathrm{H}_{2} / \mathrm{CO}_{2}$ flame with a joint velocityscalar PDF method. Reasonably good agreement with the experimental data was obtained, while the benefits in CPU time and memory storage requirements compared with the LUT and direct integration were demonstrated. Subsequently, Blasco et al. [18] employed the ANN approach to a reduced $\mathrm{CH}_{4}$ combustion mechanism including 13 species. The ANN employed involved three multilayer Perceptrons (MLPs - a type of ANN that will be described in Sec. 2.5) to compute the evolution of the reactive scalars and another one for density and temperature. A further step was taken by the same group in [19], where the Self-Organising Map (SOM - see Sec. 2.4) was adopted for clustering the data and subdividing the composition space into subdomains, while each subdomain was trained with a different MLP. The Partially Stirred Reactor (PaSR) was employed for the data generation, while the chemistry used in that study was a reduced $\mathrm{CH}_{4}$ mechanism with 9 species. The ANN results showed good agreement with the target data and great savings in CPU time and memory requirements were achieved.

In all of these works, however, the problem employed for generating the training set was the same as the one generating the set of data for testing the ANNs, and this presupposes that a similar problem must be simulated with real-time integration before the tabulated mechanism can be used. The next major challenge in applying ANNs to combustion was to obtain a representative training data set so that the resulting ANNs can be applied to real flames, without having to simulate similar flames beforehand. Chatzopoulos and Rigopoulos [20] proposed a method of training the ANNs with a data set generated by an abstract problem and then apply the ANNs to the simulation of a turbulent flame. 100 non-premixed flamelets of varying strain rates were employed to generate a data set spanning the composition space expected to be encountered in the turbulent flame. The chemistry was an RCCE-reduced mechanism involving 17 species, and the test case consisted of two $\mathrm{CH}_{4} / \mathrm{H}_{2} / \mathrm{N}_{2}$ turbulent flames (DLR-A/B). A RANS-PDF method was employed to account for the turbulence-chemistry interaction the RANS-PDF method, together with a Lagrangian stochastic method for its numerical solution. Very good agreement was obtained for the mean profiles between the ANN and RCCE results, and time savings were of the order of 100 .

While the aforementioned works employed the ANNs in order to tabulate a mechanism for use in PaSR or PDF simulations, some other studies employed ANNs in a different context. Kempf et al. [21] and Ihme et al. [22] employed ANNs with steady-state laminar flamelet to substitute the conventional tabulation in the context of Large Eddy Simulation (LES). Furthermore, in [23, 24] Sen et al. captured unsteady flameturbulence-vortex interaction effects generating an ANN database from DNS of Flame-Vortex Interaction and stand-alone Linear Eddy Mixing model calculations, respectively.

It can be concluded that, while the number of studies employing ANNs in combustion is still rather small, ANNs do represent a promising approach for dealing with the time-consuming computation of combustion chemistry, which is the major bottleneck in comprehensive modelling of turbulent combustion, particularly with PDF methods. These methods derive much of their power from the fact that they do not rely on underlying assumptions about correlation between chemical variables, but their power can only be harnessed if comprehensive chemistry is employed. Our aim is to develop a methodology where the ANNs are trained with a data set obtained via an abstract problem, and produce a model adequate for simulating a family of real combustion problems such as non-premixed turbulent flames. The present work expands and presents 
in detail the methodology first employed in [20], and subsequently couples the ANNs with an LES-PDF approach for the simulation of a turbulent flame (Sydney Flame L). This flame is a challenging test case, as it features extinction and re-ignition.

This article is structured as follows: In Sec. 2 we review the main concepts of Artificial Neural Networks and contextualize the ANNs as a tabulation method for chemical kinetics integration. Subsequently, in Sec. 3 , the methodology to generate comprehensive sample data via igniting/extinguishing flamelets is presented. In Sec. 4 we describe the ANN training methods employed in this work, present test results and compare different SOM clusterings. This is followed by the application of the ANNs to the simulation of the Sydney Flame L and comparison with real-time RCCE integration (Sec. 5) and finally by conclusions (Sec. 6).

\section{The ANN tabulation methodology}

\subsection{Overview}

ANNs are machine learning models that can be used for a variety of purposes, some of the most common of which are regression, pattern recognition, clustering and time series prediction. When employed for regression, ANNs can be used both to fit noisy data (as would arise e.g. from experimental measurements) and do approximate 'clean' data coming from a function. It is the latest function that is primarily of interest in our case. The calculation of chemical kinetics, whether by a full or reduced mechanism, amounts to the solution of a system of ODEs:

$$
\frac{d y_{i}}{d t}=f\left(y_{i}\right)
$$

where $y_{i}$ are the species mass fractions, while the right-hand side $f\left(y_{i}\right)$ is given by kinetics. The integration of this system for a fixed time step $\delta t$ implicitly defines a function, $F\left(y_{i}\right)$, that yields the mass fractions $y_{i}^{\delta t}$ for given initial conditions $y_{i}$ :

$$
y_{i}^{\delta t}=F\left(y_{i}\right)
$$

The values of this function have to be calculated by solving numerically Eqs. 1, which are a stiff system of ODEs and very expensive to solve. Therefore the basic idea of our approach is to employ an ANN to approximate this function. The ANN is a sum of non-linear basis functions involving a large number of coefficients, which are called weights and can be fitted to approximate a given function. The non-linearity of the basis functions grants them great flexibility, which is necessary in order to approximate a multidimensional problem featuring complex dynamics such as chemical kinetics.

The ANN methodology can be described by the following steps:

- Sample generation. This involves the generation of a database of results of ODE integrations for training the ANN. While ANNs have the capability to approximate complex non-linear functions very well, they effectively perform interpolation within the data set that was employed for their derivation. If they are derived from a data set obtained from a problem akin to the one being simulated, then they can perform very well, but that presupposes that we are obliged to solve our combustion problem first, 
or one similar to it, by real-time integration of the full or reduced kinetics. For the ANN tabulation to be valuable, it must be possible to derive an ANN that can cover a wide range of combustion problems, ideally by means of a data set obtained from an abstract problem (i.e. not a turbulent flame simulation). The choice of this abstract problem must be such that it covers adequately the composition space that will be visited during a simulation of the real problem. In this work we employ a set of igniting/extinguishing flamelets, as discussed in Sec. 2.2.

- Sample clustering. Combustion kinetics are multi-dimensional dynamical systems exhibiting complex non-linear behaviour. As a result, it can hardly be expected that a single machine learning tool could obtain satisfactory regression across the entire composition space. We employ clustering algorithms such as the Self-Organising Map (SOM) to generate sub-sets so that a grid of regression models can be ultimately obtained. Good clustering can substantially aid the job of the regression ANNs employed afterwards, and the process of refining the clustering map can be compared to that of refining a grid.

- ANN training. We currently employ multilayer Perceptrons (MLPs), a form of ANN, for the regression at each cluster. Training the MLP is a difficult non-linear optimisation problem, and we have so far employed the steepest descent and conjugate gradient methods. Devising an optimal strategy for utilising the big data sets generated so far is another important problem.

- Testing and application. The ANN approach will be evaluated by application to turbulent flames. The simulations will be carried out with LES and the transported probability density function (PDF) approach for modelling turbulence-chemistry interaction, while the Stochastic Fields method will be employed for the numerical solution of the PDF transport equation.

These aspects will be elaborated in the following sections.

\subsection{Sample generation via igniting/extinguishing flamelets}

Sample generation is crucial for the success of the ANN regression. In previous works $[17,18,19]$ the samples were generated via the same problem that was to be simulated. Our aim, however, is to obtain a method of generating the samples via an abstract problem, so that the resulting ANNs can be applied to a family of realistic problems. It must be stressed that any tabulation approach is by definition limited by the bounds of the dataset employed for the tabulation. ANNs are non-linear approximation models and have some capacity for extrapolation, but if they are used to predict points that lie far from their domain of validity their accuracy will of course be questionable. In this work we address this issue by constructing an abstract problem that will generate points representative of a large family of problems, in this case non-premixed flames with piloted ignition and extinction.

While it would be extremely difficult to generate a data set that covers adequately the entire multidimensional composition space defined by a mechanism comprising 15-20 species, our aim is to produce a set that covers a part that corresponds to a family of combustion problems, in this case non-premixed flames.

For this purpose, we employ a methodology based on the concept of igniting/extinguishing flamelets. By 'igniting flamelet' we mean an unsteady flamelet initialised with a very small part of the mixture fraction 
space set to equilibrium composition and the rest to unburnt, so as to emulate the effect of piloted ignition. The artificial 'pilot' in mixture fraction space is removed after a number of time steps. This concept was introduced by Chatzopoulos and Rigopoulos in [25], where it was shown that the ANNs trained via this abstract problem were able to simulate a set of turbulent non-premixed flames (DLR-A/B). In this work, in order to predict flames with significant levels of extinction such as the Sydney L, we introduce extinguishing flamelets to the generation of the training data set. These are unsteady flamelets with strain rates ranging up to twice the extinction strain rate.

A number of one-dimensional flamelet simulations are performed, with the boundary conditions being pure fuel and air. The objective is to generate a data set for training ANNs able to simulating a range of turbulent flames. The target flames ought to feature the following features in order to be compatible with this set of ANNs: they must employ the same fuel (pure or diluted), be at the same pressure and feature the same inlet temperatures. Therefore, the ANNs generated thus can simulate flames featuring both pure and diluted inlets with the same ease. The simulations are performed by solving the transient flamelet equation in mixture fraction space [26]:

$$
\rho \frac{\partial n_{j}}{\partial t}=\rho \frac{\chi(z)}{2} \frac{\partial^{2} n_{j}}{\partial z^{2}}+\dot{\omega}_{j}
$$

where $\rho$ is the density, $z$ is the mixture fraction, $n_{j}$ are the moles of species $j$ per unit mass of the mixture, $S$ is the strain rate, $\dot{\omega}_{j}$ is the reaction source term and $\chi$ is the scalar dissipation rate, given by Eq. 4 :

$$
\chi(z)=\frac{S}{\pi} e^{-2\left[\operatorname{erfc}^{-1}(2 z)\right]^{2}}
$$

This set of equations involves the mixture fraction as the independent variable and the scalar dissipation rate as a parameter. By varying these two variables, a wide range of composition states can be obtained, corresponding to flamelets ranging from equilibrium to extinction. Our approach consists of simulating a large number of flamelets for varying scalar dissipation rates and sampling composition states randomly across the mixture fraction range. Eq. 3 is a typical diffusion-reaction equation and is solved here with an in-house code employing a fractional step method, so as to decouple the reaction step and to tabulate only the latter. This is in accordance to the fractional step methods employed in RANS and LES PDF simulations. Each composition state is being integrated for a fixed time step, and the initial and final values are stored within the ANN training data set. The simulations are performed for a number of flamelets with different strain rates. In order to avoid bias in the data set, the strain rates are chosen randomly.

It must be emphasised that there is no connection between the present approach and flamelet tabulation methods. Unsteady flamelets are employed here only for sampling a set of compositions, which are then employed as initial conditions for integrating the kinetics ODEs. These compositions are thoroughly shuffled, however, before used for training. Therefore the flamelet structure is lost; it is not flamelets but individual compositions that are being used in the tabulation. The ANNs are subsequently used for tabulating the reaction step of the transported PDF calculation. The limitations of the method in terms of turbulencechemistry interaction closure are those of the transported PDF approach; there is no assumption of the burning exhibiting a flamelet regime and no implications for the Damköhler number in the flame. 


\subsection{Kinetics and mechanism reduction}

The chemical kinetics fractional step in either the flamelet or the LES-PDF (to be introduced later) formulation consists of a number of ODEs for a system comprising $N_{s}$ species and $N_{r}$ reactions:

$$
\frac{d n_{j}}{d t}=\sum_{k=1}^{N_{r}} \nu_{j k} r_{k}\left(n_{1}, \ldots, n_{N_{s}}, T, \rho\right) \quad\left(j=1, \ldots, N_{s}\right)
$$

where $r_{k}$ are the rates of the individual reactions and $\nu_{j k}$ are the stoichiometric factors. These ODEs must be complemented by the energy transport equation:

$$
\sum_{j=1}^{N_{s}} n_{j} \frac{d h_{j}^{o}}{d T} \frac{d T}{d t}=-\sum_{j=1}^{N_{s}} h_{j}^{o} \sum_{k=1}^{N_{r}} \nu_{j k} r_{k}
$$

where $h_{j}^{o}$ is the specific enthalpy of each species (including both formation and thermal), a function of temperature. As large mechanisms can easily comprise hundreds of species, a reduction of the dimensionality of this system is essential before employing the ANN tabulation. In this work we employ Rate-Controlled Constrained Equilibrium (RCCE), which is a systematic method for deriving reduced mechanisms based on time scale separation, together with a CSP-based methodology for selecting the species to be retained.

The details of the RCCE methodology have been thoroughly described in $[9,8,6,7]$ and are only summarised here. In RCCE the leading, kinetically controlled species are described from chemical kinetics without approximations, while the minimisation of free energy is employed to calculate the fast, equilibrated species by imposing a constrained equilibrium state - a state where the leading species are constrained to retain their current values. The selection of the constrained species can be based on a quantitative analysis from methods such as LOI [3, 27, 28] or CSP [9]; here we employ a CSP-based approach described in [9]. The dynamics of the major, constrained species are described via the following equation:

$$
\frac{d C_{i}}{d t}=\sum_{j=1}^{N_{s p}}\left[\alpha_{i j}^{c} \sum_{k=1}^{N_{r}} \nu_{j k} r_{k}\left(n_{1}, \cdots, n_{N_{s p}}, T, \rho\right)\right] \quad\left(i=1, \cdots, M_{c}\right)
$$

where $C_{i}$ is the concentration of the constrained species, $N_{r}$ is the total number of reactions, $\nu_{j k}$ is the stoichiometry matrix and $r_{k}$ the rate of the individual reactions. On the other hand, the fast, equilibrated species are solved through a set of algebraic equations as follows:

$$
\mu_{j}^{0}(T)+R T \ln \frac{n_{j}}{n}+R T \ln \frac{p}{p_{0}}+\sum_{i=1}^{M_{e}} \lambda_{i}^{e} \alpha_{i j}^{e}+\sum_{i=1}^{M_{c}} \lambda_{i}^{c} \alpha_{i j}^{c}=0 \quad\left(j=1, \cdots, N_{s p}\right)
$$

where $n_{j}$ is the molar concentration of species $j, n$ is the total concentration, $N_{s p}$ is the total number of species, $M_{e}$ and $M_{c}$ are the total number of elements and constraints, $\mu_{j}^{0}$ and $p_{0}$ are the chemical potential and pressure in the standard state, $\lambda_{i}^{e}$ and $\lambda_{i}^{c}$ are the element and constraint potentials and $\alpha_{i j}^{e}$ and $\alpha_{i j}^{c}$ are matrices containing the contribution of each species to each element and constraint. Eq. (8) and Eq. (7) represent the differential-algebraic formulation of RCCE. It must be noted that an equivalent implicit ODE system can be derived which is often more efficient for numerical implementation. For more information the 
reader is referred to $[6,7]$.

\subsection{SOM clustering}

It would be extremely difficult to obtain satisfactory regression of a function exhibiting as complex dynamics as a combustion mechanism does by means of a single model. Therefore we pursue an approach akin to the use of local basis functions in finite element models: rather than having a 'global' approximation, we employ a set of ANN regression models, each specialising to a part of the composition space. One question that immediately arises is how to divide the composition space into parts. With a multidimensional space where only a small part of it is covered by the data set, it would be extremely wasteful to employ a regular grid. It makes more sense to cluster points in such a way that compositions that are close to each other will be allocated to the same regression model. Accomplishing this clustering is not straightforward, however, and we would also need to have a criterion for determining the most suitable area for computing new points. In this work, the clustering is performed using the Self-Organising Map (SOM) of Kohonen [29]. The clustering of points and the use of the SOM were first introduced in the context of tabulation of combustion chemistry by Blasco et al. [19], and were also employed in an earlier study by two of the authors [20].

The SOM is a type of ANN that is employed here to identify clusters of data that are close in terms of their Euclidean distance in composition space. Each of this patterns is eventually assigned a neuron, which is effectively a composition representative of the cluster. New points can then be assigned to the neuron closest to them by a comparison of their Euclidean distance to all neurons. The neurons in a SOM map are organised in a lattice that is one- or two-dimensional in most applications. Thus, the input signal of a continuous, space of arbitrary dimensionality is transformed into a discrete one- or two-dimensional map - in this work we employ two-dimensional maps. In order to represent properly the patterns of the input space the reference map reasonably preserves the topology and the statistics of the composition space, in the sense that regions originally close to each other will remain close in the SOM and regions with high sample density on the input space will occupy large domains in the SOM. It must be emphasised that the SOM dimensions do not correspond to physical variables such as mixture fraction or progress variable. Rather, it is just a lattice on which neurons representative of parts of composition space reside. The choice of a 2D lattice is recommended by the SOM methodology - the employment of an 1D lattice is not sufficient to capture the feature of high dimensional input spaces, as was shown by Albayarak [30], and no need for employing higher-dimensional lattices has been identified.

The generation of the SOM is performed so that the reference map can subdivide the input space according to similarities in the data. The first step is based on competitive learning through similarity, which is assessed by the Euclidean distance between the input patterns and the weight vectors. The second step is based on cooperation and is regarded as the generation of the neighborhood around the winning weight vector. Finally, the third step consists of adapting and adjusting the winning weight vector and its neighbors towards the input patterns. Further details of the SOM training procedure can be found in [25]. The training is performed using the $S O M_{-} P A K$ software of Kohonen et al. [29].

One question here is how to choose the number of areas in which to split the composition space. With an increasing number of subdomains we have an increasing number of specialised MLPs, which should result in 
more refined regression. Extreme partitioning has its drawbacks, however, as too many MLPs need longer to train and furthermore it may result in areas with too few samples and uneven regression quality. Sec. 4 describes the tests of SOM partitionings that were carried out during the tabulation of the $\mathrm{CH}_{4}$ mechanism, so as to determine a partitioning exhibiting a good balance between these trends.

Before progressing, it must be mentioned that the employment of local regression MLPs may not be the only possible solution to the problem of approximating the complex dynamics of the composition space, and is also not without drawbacks. The number of subdomains is an additional parameter (termed a hyperparameter in the ANN literature) that must be determined by the methodology. Furthermore, the predictive power of the MLPs is expected to deteriorate at points lying at the boundaries between two subdomains. Finally, a computational overhead for determining the subdomain to be employed for each new point is introduced during simulation, although we have found this to be of the same order as the time needed for regression. Therefore one may ask whether the use of a single MLP should be revisited. The main drawbacks of the use of only one MLP are that it would lead to overfitting problems, due to the complexity of the function that we attend to approximate and the amount of training data required, and that it would lead to very high training times. The two approaches can be paralleled to weighted residuals modelling with global basis functions and finite element methods. Some modern approaches in the ANN literature indeed employ single networks for very large data sets, but are often employed for modelling noisy data. This is a question that we may return to in future work.

\subsection{MLP regression}

The core of the ANN chemistry tabulation approach is the regression step. This is performed with a multilayer Perceptron (MLP), a type of ANN suited to non-linear regression problems. The MLP is effectively an interpolation model that comprises a sum of non-linear basis functions, and involves a number of unknown coefficients to be determined by fitting pairs of input-output training data. MLPs have been described in detail in the ANN literature ([31], [32]) and have been used in several studies of combustion tabulation (e.g. $[17,18,19,20,25])$. In this Section we are going to give a brief account of the main concepts involved from the point of view of function approximation, which is more relevant in our context (rather than the biological analogy that is often employed in the literature).

The MLP is a non-linear mapping between two sets of variables. It is formed by a composition of nonlinear functions of a single variable that are called activation functions [32]. The model can be represented schematically by means of a network diagram, where the components of the function correspond to computing units called neurons. Fig. 1 depicts one such network with one layer of inputs and three layers of neurons. The final layer yielding the outputs is called the output layer, while the preceding layers of computing neurons (i.e. apart from the layer of inputs) are called hidden layers. In our case, the inputs are the chemical compositions and enthalpy, while the outputs are the compositions only (since we are employing the total enthalpy, which includes the formation enthalpy and is therefore not changed by reaction). Each neuron can be regarded as a basis function that performs two operations: a weighted sum of inputs from the neurons of the previous layer and the subsequent computation of a non-linear activation function. The weights are the unknown coefficients of the model, to be determined by the fitting process. The output is 


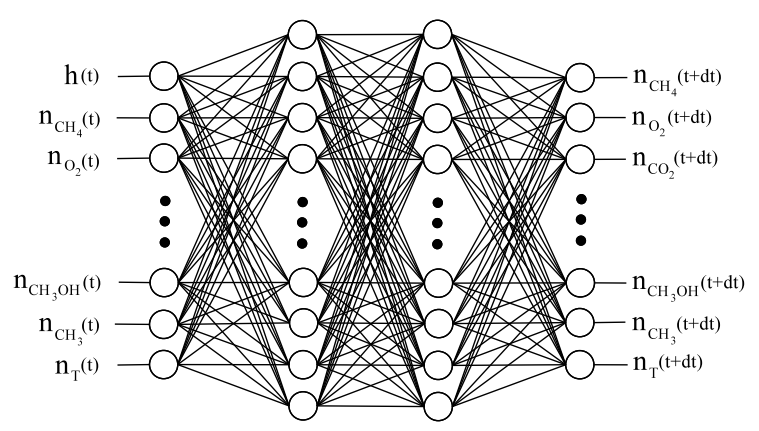

Figure 1: MLP topology

either the input value for the neurons in the next layer or the final output. For the first hidden layer, these operations can be described as follows:

$$
y_{j}^{(1)}=g\left(\sum_{i=1}^{N} w_{j i}^{(1)} x_{i}+w_{j 0}^{(1)}\right)
$$

where $x_{i}$ is the $i^{t h}$ input, $y_{j}$ is the output of the $j^{\text {th }}$ neuron and $w_{j i}^{1}$ are the weights at the first hidden layer, and $g$ is the activation function. The weight $w_{j 0}^{1}$ corresponds to the so-called 'bias neuron', which functions as a threshold and gives an affine character to the operation. The activation function should be continuous, typically in the range $(0,1)$ or $(-1,1)$, and 'activate' a neuron when its value is high. In our implementation, the activation function employed is the hyperbolic tangent, which has been found to give faster convergence [32]:

$$
g(x)=\alpha \tanh (\beta x) \quad(\alpha, \beta)>0
$$

where the values of the constants $\alpha=1.7159, \beta=2 / 3$ are suggested by Le Cun [33]. The operations at the neurons of the second hidden layer, if present, or otherwise at the output layer, are:

$$
y_{k}^{(2)}=g\left[\sum_{j=1}^{M} w_{k j}^{(2)} g\left(\sum_{i=1}^{N} w_{j i}^{(1)} y_{i}^{(1)}+w_{j 0}^{(1)}\right)+w_{j 0}^{(2)}\right]
$$

If there are more hidden layers, the operations continue likewise. It can be seen that each layer adds considerably to the complexity of the network and the number of weights. The choice of MLP topology involves setting the number of hidden layers and neurons per layer. It has a significant impact on the computation, as more complex networks are able to accommodate more complex dynamics, but one should choose the simplest possible network that is sufficient for the task required, as further complexity in the network renders both the training and simulation process slower and may result in overfitting.

The process of fitting an MLP to a set of data is called supervised learning, because input-output pairs are presented to the model in succession and the latter 'learns' from them. It is an optimisation problem, where one seeks to find the optimal values of the weights in order to minimize an objective function defined as the sum of square errors:

$$
E=0.5 \sum_{\alpha=1}^{N s} \sum_{i=1}^{N}\left(y_{i}^{\text {out }}-t_{i}^{\alpha}\right)^{2}
$$


where $E$ is the average error, $N s$ is the number of samples, $t_{i}^{\alpha}$ is the target output for sample $\alpha$ and $y_{i}^{\text {out }}$ is the MLP output.

Optimisation requires computation of the gradient of the objective function (and, in some methods, more quantities such as the Hessian). In the case of MLPs, this means calculating the change in the sum of square errors due to infinitesimal changes in the network weights. This is not a straightforward operation, however, because the implementation of MLPs is based on a neuron-per-neuron basis and the final outcome is a very complex composition of functions. The algorithm employed for this purpose is called backpropagation, and its development was pivotal in the history of ANNs. It effectively consists of a chain of partial differentiations, and one is referred to $[32,31]$ for more details. The most straightforward optimisation method that can be employed is steepest descent. In our implementation we employ the conjugate gradient method, due to the fact that it is efficient for large networks such as the ones employed in this study and free of adjustable parameters. The method uses a line search technique along with a vector that points towards the minima to compute the new weights. The conjugate gradient method was first used for ANN training for combustion tabulation in [18], and was also employed in an earlier study by two of the authors [20].

The training procedure employs the cross-validation technique [31]: the training data set assigned to each MLP is split into a training set and one used for testing that takes place during the training process. Apart from offering a preliminary evaluation of the MLP's performance, cross-validation is also used as a stopping criterion for the training: if the error has not been decreasing for 50 iterations consecutively, the training process is stopped.

A separate MLP must be trained for each SOM subdomain. The training process is carried out with an inhouse developed code, which trains the MLPs in parallel. Finally, the MLP's regression ability must be tested by computing a new set of data and comparing the output with that of the function being approximated, in this case the RCCE result. For this reason, one part of the data collected is reserved for testing purposes. The SOM-MLP synergy is shown schematically in Fig. 2.

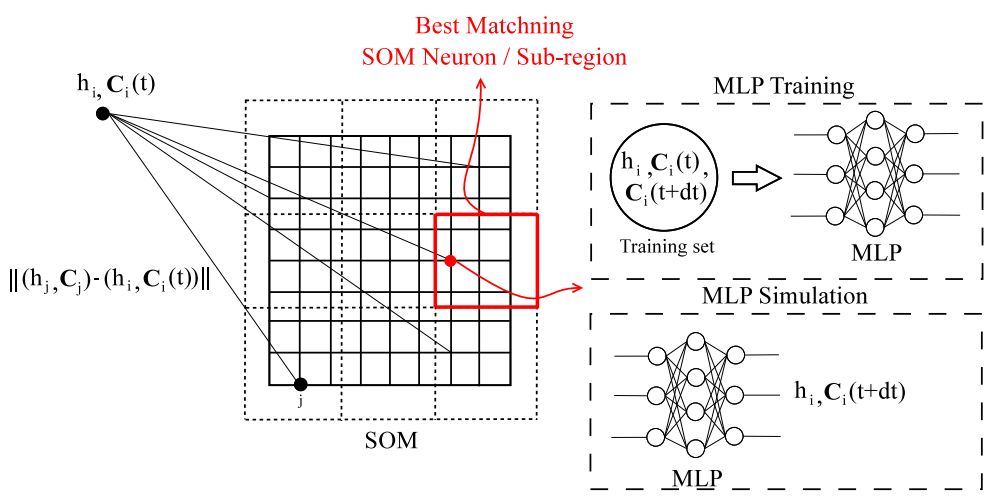

Figure 2: Schematic SOM-MLP topology. An input vector is presented to the SOM, and the best subdomain is chosen. The dashed lines indicate the boundaries of subdomains, which may include several SOM neurons. Subsequently, the corresponding MLP is employed for its computation. 


\section{Coupling ANNs with LES-PDF modelling of turbulent combustion}

\subsection{The LES-PDF equations}

The LES-PDF method is adopted for the simulation of the flow field and turbulence-chemistry interaction. This method has been extensively described in e.g. [34, 35, 36]; its main advantages are that it captures accurately the details of the flow field, while at the same time the reaction source term appears in closed form. The main closure problem in a transported PDF method is related to the micromixing term, but the influence of the micromixing model in an LES-PDF simulation is far less than in a RANS-PDF.

The LES-PDF equations can be obtained by applying a spatial filter - in our case a 'box' filter - to the instantaneous transport equations for reacting flows (see e.g. [37]). The resulting equations are:

$$
\begin{gathered}
\frac{\partial \bar{\rho}}{\partial t}+\frac{\partial \bar{\rho} \tilde{u}_{i}}{\partial x_{i}}=0 \\
\frac{\partial \bar{\rho} \tilde{u}_{i}}{\partial t}+\frac{\partial \bar{\rho} \tilde{u}_{j} \tilde{u}_{i}}{\partial x_{j}}=\frac{\partial \bar{p}}{\partial x_{i}}+\frac{\partial}{\partial x_{j}}\left[2 \mu \tilde{S_{i j}}-\frac{2}{3} \mu \tilde{\left.S_{k k} \delta_{i j}\right]}+\frac{\partial \tau_{i j}^{s g s}}{\partial x_{j}}+\bar{\rho} g_{i}\right. \\
\frac{\partial \bar{\rho} \tilde{\phi_{\alpha}}}{\partial t}+\frac{\partial \bar{\rho} \tilde{u}_{i} \tilde{\phi_{\alpha}}}{\partial x_{i}}=\frac{\partial}{\partial x_{i}}\left[\bar{\rho} D \frac{\partial \tilde{\phi_{\alpha}}}{\partial x_{i}}\right]-\frac{\partial J_{i, \alpha}^{s g s}}{\partial x_{i}}+\overline{\rho \dot{\omega}_{\alpha}}
\end{gathered}
$$

where $\tilde{u}$ is the Favre-averaged (i.e. density averaged) filtered velocity, $\bar{p}$ is the mean pressure, $\mu$ is the viscosity, $\tilde{S_{i j}}$ is the resolved rate of strain tensor, $\tau_{i j}^{s g s}$ is the sub-grid Reynolds stress tensor, $\bar{\rho} g_{i}$ is the gravitational force, $\tilde{\phi}_{\alpha}$ is the Favre-average filtered reactive scalar, $D$ is the diffusion coefficient, $J_{i, \alpha}^{s g s}$ is the sub-grid scalar flux and $\overline{\dot{\omega}_{\alpha}}$ is the source term. The last one is a function of the joint-scalar pdf, and therefore appears in closed form. The Smagorinsky model [38] is employed here for modelling the sub-grid stresses.

The sub-grid probability density function is introduced in order to obtain closure for the non-linear reaction source terms. The evolution of density-weighted joint sgs-pdf $\tilde{P}$ is described by the following equation:

$$
\bar{\rho} \frac{\partial \tilde{P}}{\partial t}+\bar{\rho} \tilde{u}_{i} \frac{\partial \tilde{P}}{\partial x_{i}}-\sum_{\alpha=1}^{N_{s p}} \frac{\partial \bar{\rho} \dot{\omega}_{\alpha} \tilde{P}}{\partial \psi_{\alpha}}=-\frac{\partial}{\partial x_{i}}\left[\left(\frac{\mu}{\sigma}+\frac{\mu_{s g s}}{\sigma_{s g s}}\right) \frac{\partial \tilde{P}}{\partial x_{i}}\right]-\frac{\bar{\rho}}{2 \tau_{s g s}} \sum_{\alpha=1}^{N_{s p}}\left[\frac{\partial\left(\psi_{\alpha}-\tilde{\phi}_{\alpha}\right)}{\partial \psi_{\alpha}} \tilde{P}\right]
$$

where $\psi_{\alpha}$ is the sample space variable corresponding to species $\phi_{\alpha}, \sigma$ is the Prandtl number and $\tau_{s g s}$ is the sub grid time scale, $\tilde{\phi}$ is the Favre-average of the random field and $\mu_{\text {sgs }}$ is the sub-grid viscosity. The Interaction via Exchange with the Mean (IEM) or Linear Mean Square Estimation (LMSE) [39, 40] is employed for the closure of the micromixing term, with the standard value of 2 for the micromixing constant. The IEM is employed, in spite of its shortcomings in terms of physically describing the mixing of reacting scalars, primarily due to its suitability for combining with the stochastic fields method that will be used for numerical solution of the PDF transport equation; furthermore, as mentioned above, the influence of the micromixing model in LES-PDF is diminished as compared to RANS-PDF. 


\subsection{The Stochastic Fields method}

As eq. 16 is multidimensional, its numerical solution can be obtained more efficiently by stochastic methods, where the sample paths of an equivalent system of Stochastic Partial Differential Equations (SPDEs) are calculated in order to approximate the pdf. These methods were pioneered in the context of RANS-PDF methods, and have been reviewed extensively in [41, 42]. Out of three different approaches that were proposed in the context of RANS, two have been employed in LES: the Lagrangian particle method and the stochastic fields. In this work we employ the stochastic fields which offers several advantages, particularly when coupled with LES, such as the ability to obtain a smooth solution even when only a few fields are employed. Two approaches can be adopted to derive the SPDEs for the stochastic fields, the Ito [43] and Stratonovich [44] formulation of the stochastic integral. In this work we employ the Ito interpretation. More details about this formulation are available in $[35,36,37,45,46]$. The stochastic differential equation yielding the evolution of each stochastic field is shown below:

$$
\bar{\rho} d \xi_{\alpha}^{n}=-\bar{\rho} \tilde{u} \frac{\partial \xi_{\alpha}^{n}}{\partial x_{i}} d t+\bar{\rho} \dot{\omega}_{\alpha} d t+\frac{\partial}{\partial x_{i}}\left(\Gamma^{\prime} \frac{\partial \xi_{\alpha}^{n}}{\partial x_{i}}\right) d t-\frac{\bar{\rho}}{2 \tau_{s g s}}\left[\xi_{\alpha}^{n}-\tilde{\phi_{\alpha}}\right] d t+\bar{\rho} \sqrt{\frac{2 \Gamma^{\prime}}{\bar{\rho}}} \frac{d \xi_{\alpha}^{n}}{d x_{i}} d W_{i}
$$

where $\Gamma^{\prime}$ is the total diffusion coefficient, $\xi_{\alpha}^{n}$ is the stochastic field and $d W_{i}(t)$ is the increment of the Wiener process. The mean of each species is approximated from the sample paths, $\xi_{\alpha}^{n}$, as follows:

$$
\tilde{\phi}_{\alpha}=\frac{1}{N} \sum_{n=1}^{N} \xi_{\alpha}^{n}
$$

The reaction source term in each stochastic field is evaluated via the ANNs. A flowchart of the implementation of the LES-PDF-ANN methodology is shown in Fig. 3.

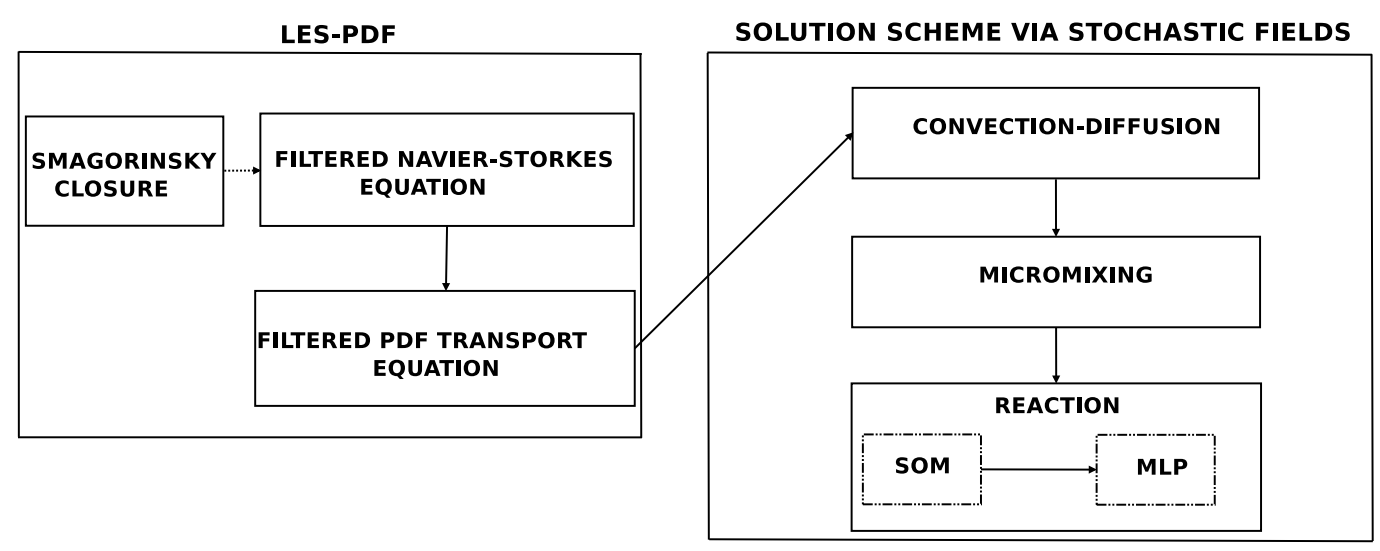

Figure 3: Schematic LES-PDF-ANN representation with stochastic fields solution scheme

\section{ANN tabulation of an RCCE-reduced mechanism for $\mathrm{CH}_{4}$ combustion}

In this section, the methodology described above will be applied to the tabulation of an RCCE-reduced $\mathrm{CH}_{4}$ combustion mechanism, itself derived via a CSP-based methodology from the detailed mechanism GRI 1.2 
[47]. The methodology and the mechanism are discussed in detail in [9], where it is also shown that the predictions of the RCCE-reduced mechanism are in excellent agreement with the detailed mechanism over a wide range of problems including flamelets with varying strain rates, premixed flames and ignition. The following 16 species are retained as RCCE constraints in the mechanism: $\mathrm{H}_{2} \mathrm{O}, \mathrm{CO}_{2}, \mathrm{CH}_{4}, \mathrm{CO}, \mathrm{O}_{2}, \mathrm{H}_{2}$, $\mathrm{C}_{2} \mathrm{H}_{2}, \mathrm{C}_{2} \mathrm{H}_{4}, \mathrm{CH}_{3}, \mathrm{C}_{2} \mathrm{H}_{6}, \mathrm{OH}, \mathrm{O}, \mathrm{CH}_{2} \mathrm{O}, \mathrm{CH}_{2} \mathrm{CO}, \mathrm{CH}_{3} \mathrm{OH}$ and $\mathrm{H}$.

As described in Sec. 2.2, the first step of the methodology is to generate the data set by means of flamelet simulations. 120 flamelets were computed with strain rate varying randomly, from $1 \mathrm{~s}^{-1}$ up to $1100 \mathrm{~s}^{-1}$, which is about twice the extinction strain rate for $\mathrm{CH}_{4}$-air combustion. The reason for employing random strain rates was to avoid bias in the data set. The flamelets were computed with 500 grid points in the mixture fraction space, from their initial condition up to the steady state, with a time step of $10^{-6} \mathrm{~s}$. The pilot was activated around the stoichiometric mixture fraction with limits randomly chosen at each simulation, and was maintained for 500 steps. Fig. 4 displays the temperature and the $\mathrm{CO}_{2}$ mass fraction of a flamelet with $400 \mathrm{~s}^{-1}$ strain rate, i.e. close to extinction, together with a curve indicating the pilot.
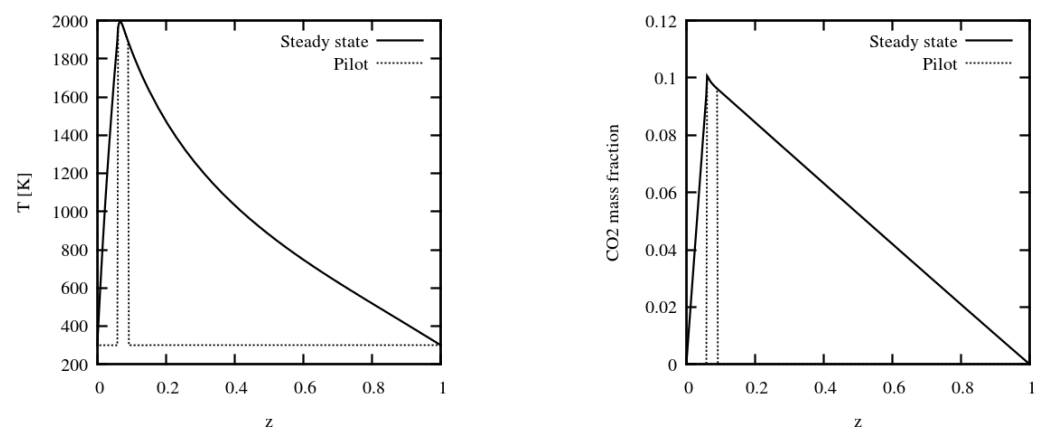

Figure 4: Profiles of flamelets ignited from pilot for Temperature and $\mathrm{CO}_{2}$

The next step is the generation of the SOM. The map consists of a set of vectors in composition space with values normalised in the $(-1,1)$ range, each of them representing one area for which a specialised MLP is to be trained. Fig. 5 show some individual components of the vectors in the two-dimensional 20-20 SOM lattice. It can be seen that points having similar values remain close to each other. A well-trained SOM that performs good clustering should feature smooth changes in the components. Furthermore, it can be seen that different areas of the SOM represent areas with common qualitative features of the composition space, such as areas with burnt or unburnt mixture that can be identified e.g. by having higher or lower $\mathrm{CO}_{2}$ concentration.

Fig. 6 shows the composition of the SOM neurons superimposed on the composition space accessed by the training set. It must be stressed that these are projections of the points in the higher-dimensional composition space onto two-dimensional subspaces such as $\mathrm{CO}-\mathrm{CO}_{2}$. Therefore, points being close in these projections are not necessarily close in terms of Euclidean distance in composition space. However, it can still be seen that the denser regions of these projections are allocated more SOM vectors. This is also a feature expected from a good map, as it means that regions that are accessed more will be accommodated by more specialised MLPs. 

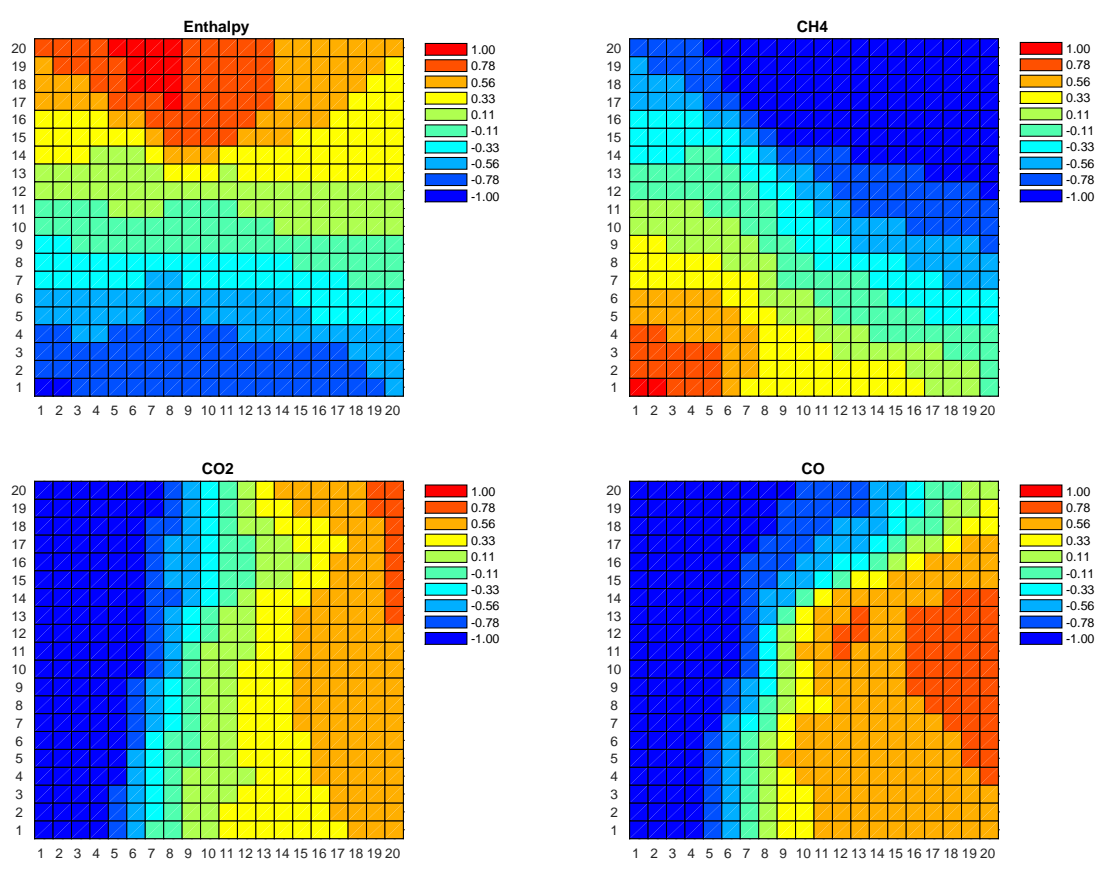

Figure 5: SOM map for enthalpy, $\mathrm{CH}_{4}, \mathrm{CO}_{2}$ and $\mathrm{CO}$. The values shown are the magnitudes of the $\mathrm{SOM}$ map vector components (values are normalised in the $(-1,1)$ range).

Having obtained the SOM vectors, the training set was allocated to the SOM areas and the MLP training process was carried out. $60 \%$ of the samples in each subdomain were used for training, while the other $40 \%$ were employed for cross-validation. The final set of MLPs was tested with yet another data set, comprising data spanning the whole composition space and never used during the training process. This data set was generated with a new set of flamelets featuring different (also randomly selected) strain rates, and comprised 1,200,000 samples (758 MB). The data were projected to the SOM subdomains and subsequently calculated by the corresponding MLPs. This is the ultimate test of the entire SOM-MLP tabulation, and it also serves to compare different SOM partitionings. Three different SOM-MLP topologies were trained and tested with unseen data, and results are compared in terms of average RMS error (Table 1). The results show that the maps using 400 and 100 subdomains exhibited lower error than the one with 25 SOM subdomains. The difference between these two was very small, indicating that there was no need for further refinement of the SOM. Also, further partitioning can result in subdomains with a very small number of samples, thus compromising the quality of the fitting of the corresponding MLP. Fig. 7 show a comparison between RCCE and ANN for the unseen data, using the 400 subdomains SOM-MLP, and it can be seen that the ANN predictions are very accurate and exhibit very few outliers. 

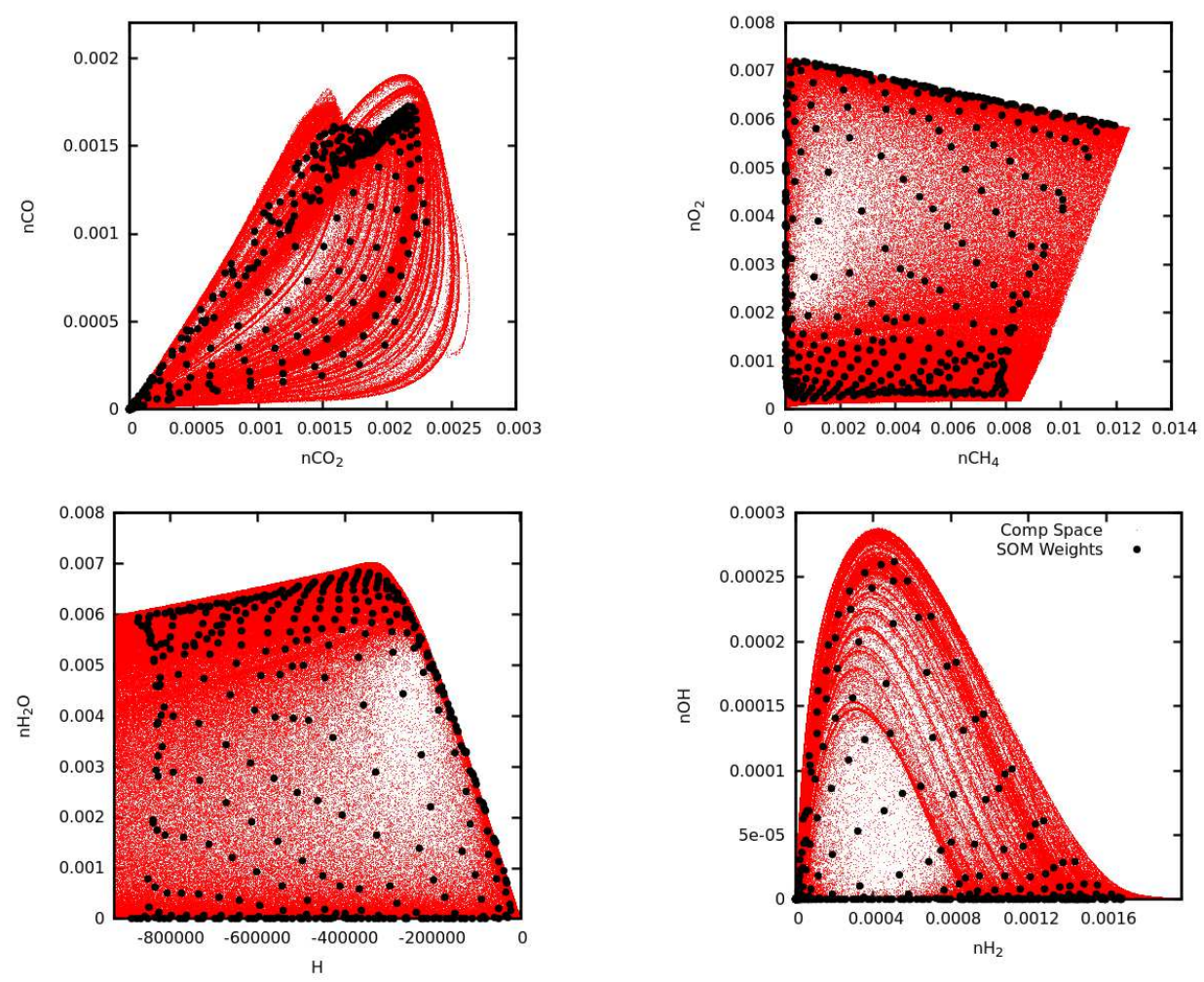

Figure 6: Composition points accessed by the training set and SOM weight vectors $(\mathrm{kmol} / \mathrm{kg})$ for $\mathrm{CO}-\mathrm{CO}_{2}, \mathrm{CH}_{4}-\mathrm{O}_{2}$, Enthalpy $-\mathrm{H}_{2} \mathrm{O}$ and $\mathrm{H}_{2}-\mathrm{OH}$

\section{Application of the ANN methodology to the Sydney Flame L}

\subsection{Review of previous work}

In this section, the mechanism developed in Sec. 4 will be applied to a simulation of the Sydney flame $\mathrm{L}$ studied by Masri et al. in [48, 49], via the LES-PDF approach and stochastic fields method described in Secs. 3.1 and 3.2. This flame has already been the subject of several numerical studies, which will be briefly reviewed below.

A first group of works involving RANS includes [50, 51, 52]. The study of Chen et al. [50] adopted RANS coupled with a joint-scalar transported PDF, using the Curl model as a closure for the micromixing term and a Monte Carlo solution technique based on Lagrangian particles, combined with two different approaches for the chemical kinetics: a reduced mechanism using four species and five reaction steps and a four-scalar constrained equilibrium model. Mean mass fraction profiles of both reduced mechanism and the constrained model show reasonably good agreement with the experiment, considering the method employed. However, the simulation does not capture well the experimental spreading rate and peak value at any of the crosssections. These shortcomings can be attributed both to the micromixing model and to the simplified kinetic schemes employed. The work of Masri and Pope [51] employed RANS coupled with a joint-scalar-velocity PDF; the latter was solved through Monte Carlo simulation. The mean radial profiles were reasonably 


\begin{tabular}{|c|c|c|c|}
\hline SOM subdomains & 25 & 100 & 400 \\
\hline $\mathrm{CH}_{4}$ & $0.270 \mathrm{E}-3$ & $0.477 \mathrm{E}-5$ & $0.607 \mathrm{E}-5$ \\
$\mathrm{O}_{2}$ & $0.990 \mathrm{E}-4$ & $0.189 \mathrm{E}-5$ & $0.225 \mathrm{E}-5$ \\
$\mathrm{H}_{2} \mathrm{O}$ & $0.890 \mathrm{E}-4$ & $0.185 \mathrm{E}-5$ & $0.213 \mathrm{E}-5$ \\
$\mathrm{CO}$ & $0.350 \mathrm{E}-4$ & $0.745 \mathrm{E}-6$ & $0.115 \mathrm{E}-5$ \\
$\mathrm{CO}_{2}$ & $0.396 \mathrm{E}-4$ & $0.100 \mathrm{E}-5$ & $0.119 \mathrm{E}-5$ \\
$\mathrm{H}_{2}$ & $0.544 \mathrm{E}-4$ & $0.445 \mathrm{E}-6$ & $0567 \mathrm{E}-6$ \\
$\mathrm{H}$ & $0.216 \mathrm{E}-5$ & $0.104 \mathrm{E}-6$ & $0.136 \mathrm{E}-6$ \\
$\mathrm{O}$ & $0.148 \mathrm{E}-6$ & $0.130 \mathrm{E}-6$ & $0.149 \mathrm{E}-6$ \\
$\mathrm{OH}$ & $0.979 \mathrm{E}-6$ & $0.137 \mathrm{E}-6$ & $0.175 \mathrm{E}-6$ \\
$\mathrm{C}_{2} \mathrm{H}_{2}$ & $0.102 \mathrm{E}-4$ & $0.721 \mathrm{E}-7$ & $0.744 \mathrm{E}-7$ \\
$\mathrm{C}_{2} \mathrm{H}_{4}$ & $0.435 \mathrm{E}-5$ & $0.382 \mathrm{E}-7$ & $0.366 \mathrm{E}-7$ \\
$\mathrm{C}_{2} \mathrm{H}_{6}$ & $0.210 \mathrm{E}-5$ & $0.271 \mathrm{E}-7$ & $0.309 \mathrm{E}-7$ \\
$\mathrm{CH}_{3} \mathrm{OH}$ & $0.403 \mathrm{E}-7$ & $0.487 \mathrm{E}-8$ & $0.798 \mathrm{E}-8$ \\
$\mathrm{CH}_{2} \mathrm{O}$ & $0.276 \mathrm{E}-5$ & $0.935 \mathrm{E}-7$ & $0.108 \mathrm{E}-6$ \\
$\mathrm{CHO}_{3}$ & $0.439 \mathrm{E}-5$ & $0.743 \mathrm{E}-7$ & $0.107 \mathrm{E}-6$ \\
$\mathrm{CH}_{2} \mathrm{CO}$ & $0.403 \mathrm{E}-7$ & $0.275 \mathrm{E}-8$ & $0.277 \mathrm{E}-8$ \\
\hline Average rms & $0.362 \mathrm{E}-4$ & $0.671 \mathrm{E}-6$ & $0.836 \mathrm{E}-6$ \\
\hline
\end{tabular}

Table 1: Testing rms error per species for different combinations of SOM subdomains.
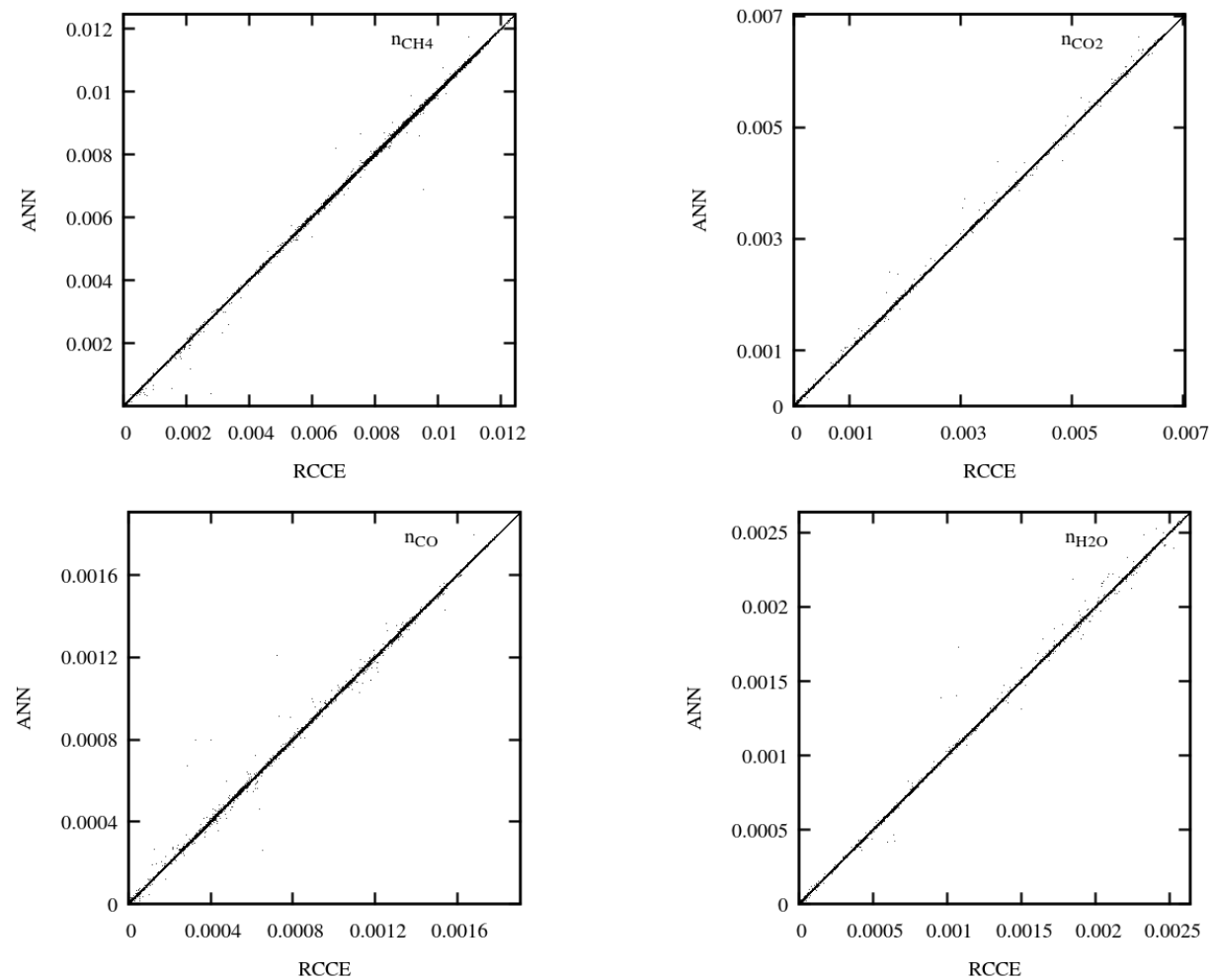

Figure 7: Comparison of SOM-MLP prediction against RCCE target values for unseen data (approximately 1.2 million points) 
predicted at $x / D=20$ but largely overpredicted at $x / D=30$. The researchers concluded that the results suffered from the simplified thermochemical approaches employed. Third, the work of Jones et al. [52] employed a joint scalar PDF approach with two micromixing models, Curl and IEM. The Eulerian stochastic fields formulation was used to solve the PDF transport equation, employing a 4-step global hydrocarbon mechanism. Mean radial profile results close to the nozzle are reasonably in agreement with the experiment, but not very good at the sections downstream. Overall, the discrepancies in this group of studies can be expected given that, in the era of RANS simulations, the limitations in CPU time meant that only highly simplified mechanisms could be employed.

So far, few works have employed LES for the simulation of the Sydney flames. The work of Prasad et al. [53] employed the LES-PDF and stochastic fields formulation, in conjunction with a reduced mechanism including 19 species, to the Sydney flames L, B and M. The results were in very good agreement with the experiments, but the computational demands were very high (1600 CPUs for 2 days), as can be expected from a simulation employing the LES-PDF method with a mechanism of this level of detail and a fine grid (approximately 7.2M cells). Lastly, the work of Wang et al. [54] was based on the application of the ISAT method in the context of LES-PDF approaches with Lagrangian particles to simulate the transient behaviour of Sydney flames L,B and M. The mean radial profile results were in reasonable agreement with the experiment, but the computational demands were still considerable (about 150,000 CPU hours for a grid comprising 746496 cells and 30 stochastic particles per cell). It must be acknowledged that these studies were conducted with earlier CPUs, and that any LES-PDF approach is going to incur a considerable computational cost (even for computing the flow field alone). Therefore, in the present study the performance of ANNs will be evaluated by means of a) the speed-up factor as compared to the integration of the reduced mechanism, and b) the proportion of each time step in the LES-PDF simulation taken by the ANNs. The latter is a good indication of the efficiency of the method, as the best one can do with a tabulation approach is to bring down this proportion.

\subsection{Numerical setup}

The experimental setup for the Sydney flame L features a burner identical to the Sandia burner with a main fuel jet of $D=7.2 \mathrm{~mm}$ and a pilot annulus with diameter of $D_{p}=18 \mathrm{~mm}$. The bulk velocity of the fuel jet is $U_{J}=41 \mathrm{~m} / \mathrm{s}$, resulting in Reynolds number $R e=20500$, while the bulk velocity of the unburned pilot is $U_{u p}=3 \mathrm{~m} / \mathrm{s}$. The pilot mixture fraction is stoichiometric $\left(Z_{p}=0.055\right)$. The velocity of the fully burnt pilot is estimated to be $U_{u p}=24 \mathrm{~m} / \mathrm{s}$. A co-flow comprising air with velocity of $U_{c f}=15 \mathrm{~m} / \mathrm{s}$ is maintained around the burner. The Eulerian flow field is computed by the in-house CFD code LES-BOFFIN using a domain with dimensions $39.6 \times 12 \times 12 \mathrm{~cm}$, via a Cartesian finite volume grid of $300 \times 96 \times 96$. An ensemble of 8 stochastic fields is used for approximating the solution of the PDF transport equation. This number has been used in several previous studies $([36,45,46,53])$ and has been found to be adequate, at least for predicting mean statistics.

\subsection{Results and discussion}

The LES-PDF-RCCE simulations where performed on the ARCHER UK National Supercomputing Service, employing 80 processors. The LES-PDF-ANN simulations were fast enough to be carried out on a work- 


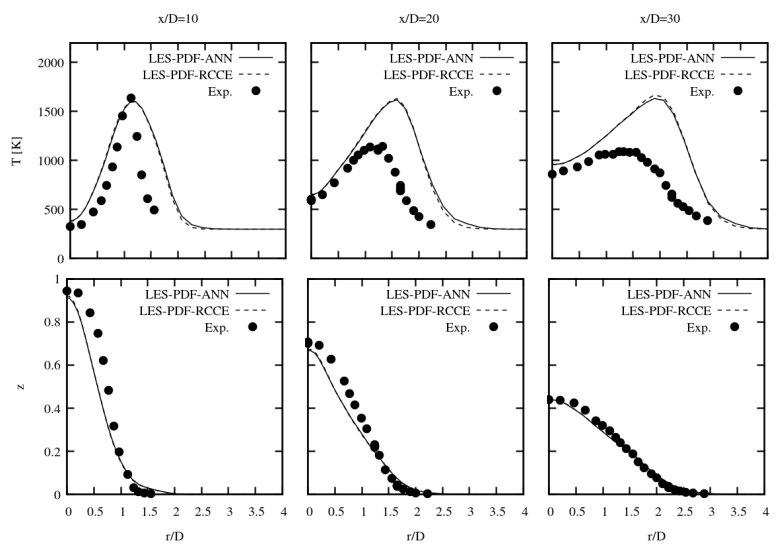

Figure 8: LES-PDF-ANN and LES-PDF-RCCE mean temperature and mixture fraction radial profiles

station, but for comparison purposes they were also run on ARCHER and the average time per time step was recorded. The ANN-MLP employed was the one found to be optimal after the tests described in Sec. 4, comprising 400 SOM subdomains and an MLP topology of 2 hidden layers with 30 neurons each.

Mean and rms temperature and mixture fraction profiles are shown in Fig. 8 and 9. The most important comparison here is between the RCCE and ANN results, as the objective of the latter is to approximate the former, while the agreement with experiments is subject to further factors associated with the LES and micromixing modelling. We can see that the profiles acquired through the ANN simulation reproduce very accurately the outcome given by the RCCE simulation on the three instances $(x / D=10, x / D=20$ and $x / D=30)$; in most cases, the curves are indistinguishable. The rms are more difficult to predict, and small discrepancies can be noticed in their profiles, although the agreement is still quite good.

Compared with the experimental results, we can see that the models reproduce quite well the variation of the mixture fraction at all cross-sections. The temperature is well predicted at the first cross-section, but considerably overpredicted at the other two. Profiles of $\mathrm{CH}_{4}, \mathrm{CO}_{2}, \mathrm{CO}, \mathrm{H}_{2} \mathrm{O}, \mathrm{H}_{2}$ and $\mathrm{O}_{2}$ mean and rms mass fraction are seen in Fig. 10 and 11. The prediction of all reactive scalars shows good agreement with the experiments close to the nozzle but deviates downstream, in accordance with the temperature predictions. $\mathrm{CO}$ is harder to predict, and its profiles show reasonable agreement at $x / D=10, x / D=20$ but deviate considerably at $x / D=30$. It must be noted that the RCCE-reduced mechanism has been shown to be very accurate compared with direct integration in [9] where it was derived. Also, a very similar RCCEreduced $\mathrm{CH}_{4}$ mechanism, comprising 18 constraints and based on the GRI 3.0 mechanism, was employed in [55] to simulate the Sandia series of flames and produced results very close to direct integration of the full mechanism. Therefore, the chemistry can be regarded as very detailed and the discrepancies between the simulations and experiments can be attributed mainly to the grid and micromixing model (IEM). The ANN simulation is also able to capture the local extinction and re-ignition, phenomena that exist to a large extent in this flame. The contour plot of the flame is shown in the Fig. 12, where the black line indicates stoichiometric mixture fraction $(z=0.055)$.

Although the main focus of this paper is on the comparison between the RCCE and its approximation via ANNs, it is worthwhile to compare the results with the previous LES simulations of this flame. Compared 


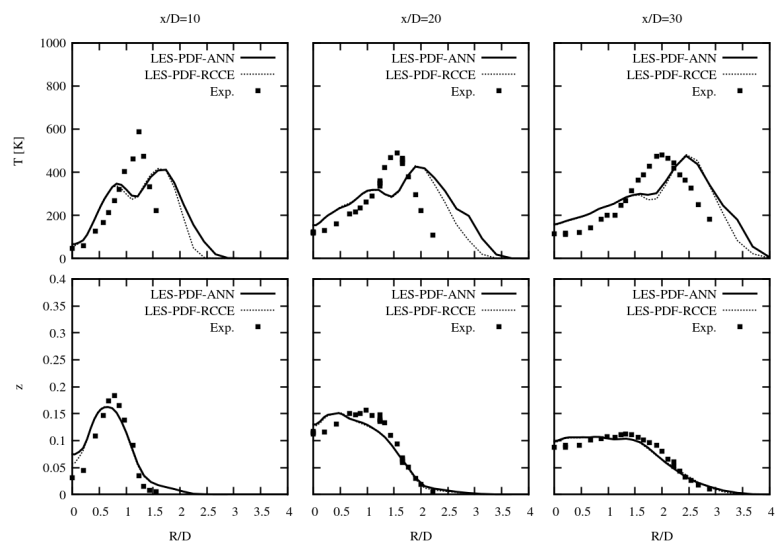

Figure 9: LES-PDF-ANN and LES-PDF-RCCE rms temperature and mixture fraction radial profiles

with the LES-PDF study of Wang et al. [54], the results are better in some respects and worse in others - in particular the spreading rate in the mean temperature and $\mathrm{CO}_{2}$ profiles is better predicted here, but Wang et al. capture better the peak values. The mean $C O$ mass fraction profile is also better predicted here. The other LES-PDF study of this flame, due to Prasad et al. [53], obtained better results than the present study with only small discrepancies from the experimental results. This is likely to be due to the use of a body-fitted pyramid grid that follows better the shape of the flame.

The CPU time measurements (Table 2) indicate that the ANN tabulation has very modest CPU time requirements, about two orders of magnitude faster than the real-time RCCE integration. More important is the fact that the ANN fractional step takes on average 4.6 times less CPU time than the convection-diffusion fractional step, thus indicating that chemistry is no longer the bottleneck in the LES-PDF simulation. Finally, the disk storage space required for the ANNs (i.e. the weights) is only $36 \mathrm{MB}$. These modest requirements mean that the LES-PDF simulation of a turbulent flame is feasible even on a workstation, and the bottleneck is now the grid and the level of detail on the resolution of the flow structures required from the LES. It must be mentioned that, in transported PDF methods, the real-time integration of a mechanism (whether full or reduced) is always expensive and typically takes up the majority of the CPU time of the calculation. Even if this proportion could be brought down somewhat with a faster mechanism, $\mathrm{CH}_{4}$ is one of the simplest fuels; more complex fuels require very large and stiff mechanisms. Currently LES-PDF simulations are very expensive even with simple fuels and test cases such as jet flames, hence the long CPU times employed by the other LES-PDF simulations of this flame (as noted in the previous point), and become virtually unfeasible with complex fuels. Our main objective in developing this approach is to pave the way for transported PDF simulations which, although computationally heavy, offer advantages due to not presuming correlations between species. 

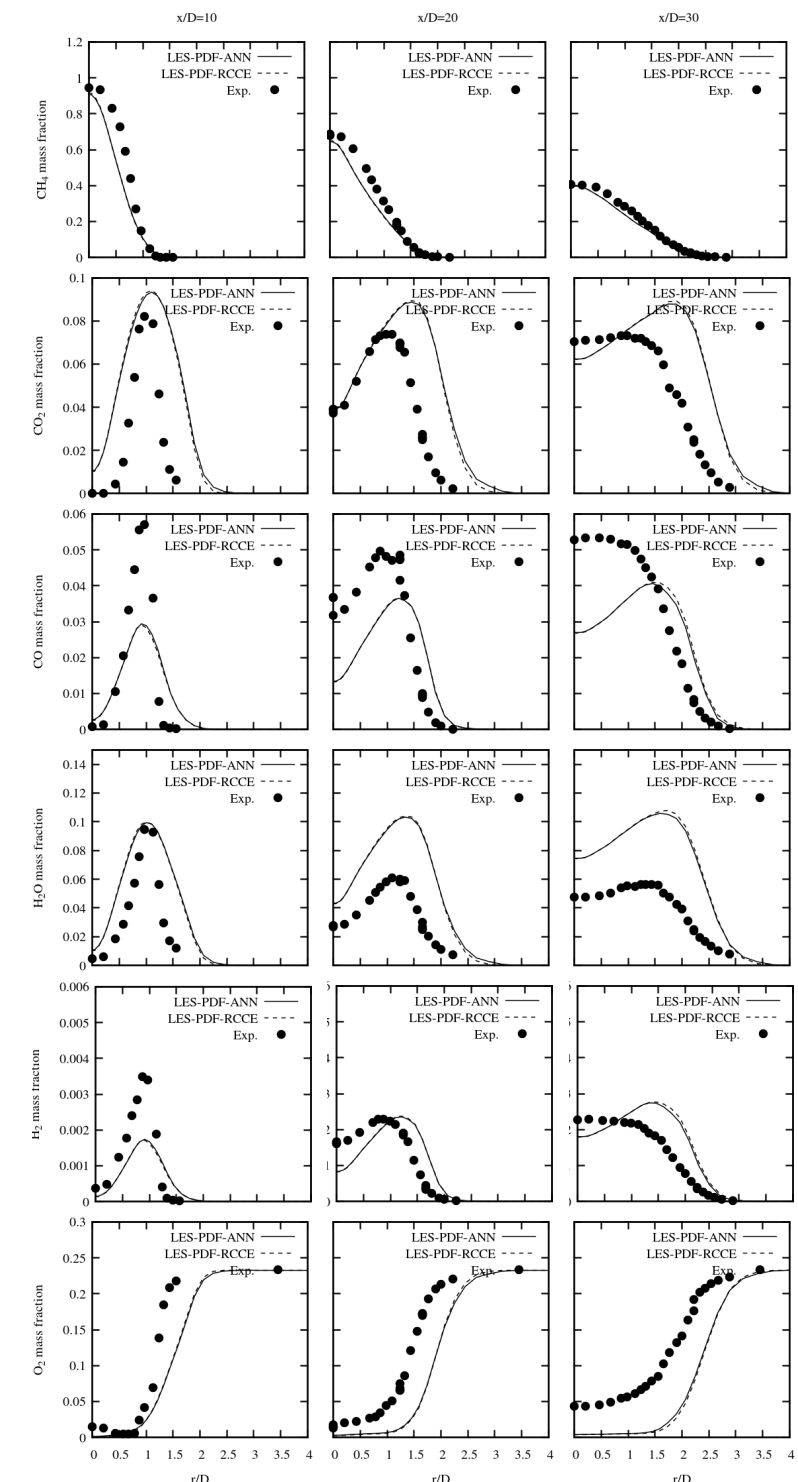

Figure 10: Radial profiles of mean reactive scalars: comparison of experiments, LES-PDF-RCCE and LES-PDF-ANN

\begin{tabular}{|c|c|c|}
\hline Method & Convection-Diffusion (s) & Reaction (s) \\
\hline RCCE & 7 & 125 \\
\hline ANN & 7 & 1.5 \\
\hline
\end{tabular}

Table 2: Average CPU time for ANN and RCCE real time integrator 

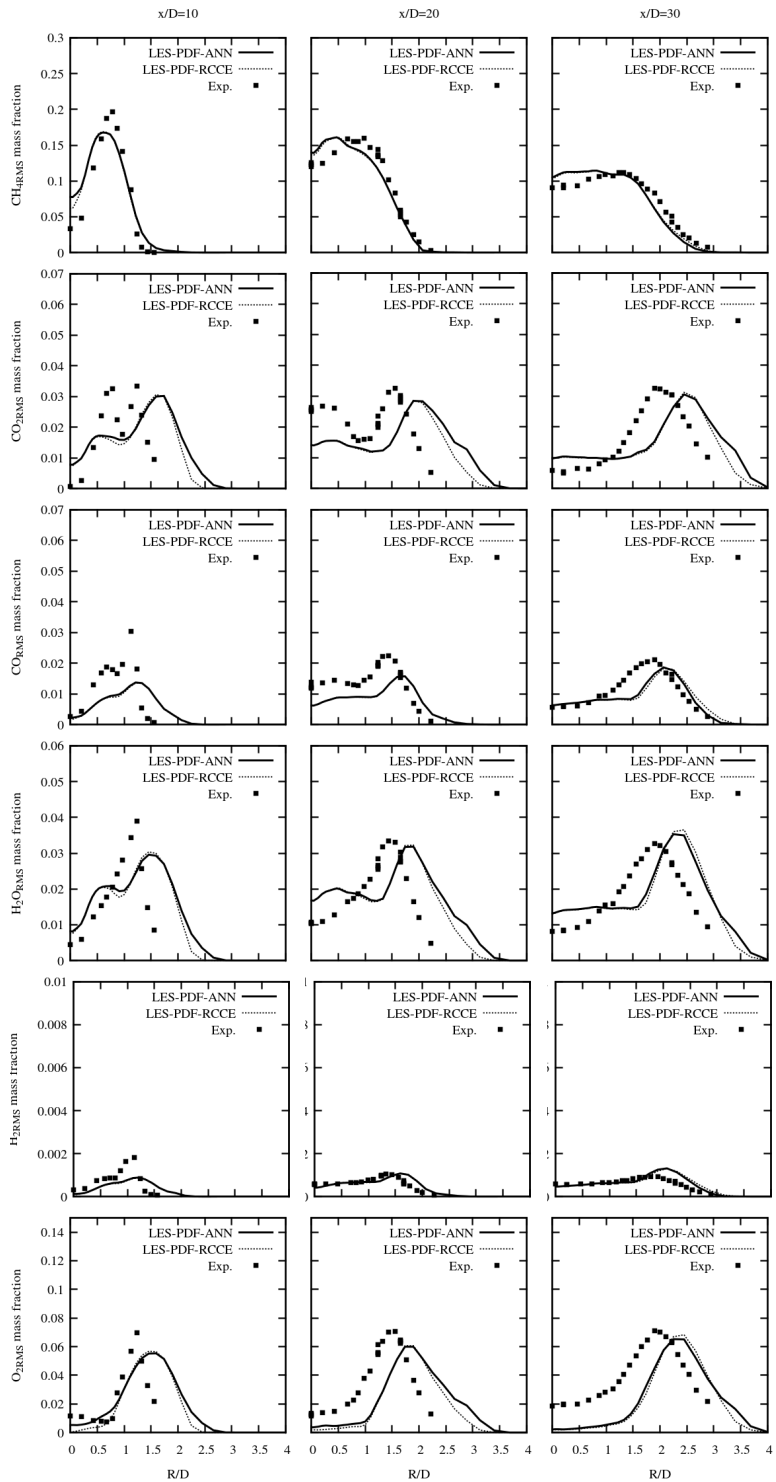

Figure 11: Radial profiles of rms of reactive scalars: comparison of experiments, LES-PDF-RCCE and LES-PDF-ANN 


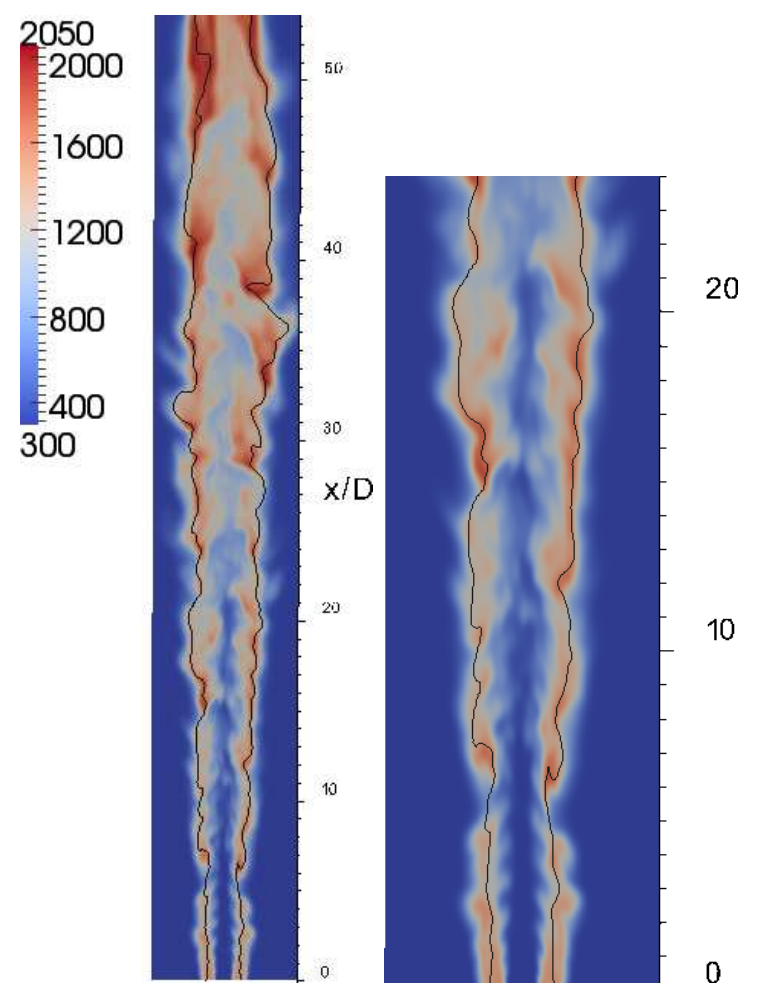

Figure 12: Contour plots of Sydney flame L computed with LES-PDF-ANN: a) full domain b) zoom on the middle section of the flame

\section{Conclusions}

In this work we presented a methodology for tabulation of combustion chemistry via ANNs. Coupled with a systematic reduction method (RCCE) and timescale analysis (CSP), the methodology forms part of a comprehensive approach for developing tabulated combustion mechanisms suitable for computationally intensive combustion simulations, such as those employing PDF methods and complex fuels. An important feature of the methodology is the generation of a sample space for training the ANNs via an abstract problem - the igniting/extinguishing flamelets - in order to cover the part of composition space anticipated to be visited by a family of combustion problems, in this case turbulent non-premixed flames with local extinction and re-ignition. The method was subsequently employed to tabulate an RCCE-reduced mechanism of $\mathrm{CH}_{4}$ combustion, based on GRI 1.2 and derived by CSP analysis that has been presented and validated in [9]. Subsequently, the mechanism was employed within an LES-PDF approach solved numerically via the Stochastic Fields method, in order to predict a non-premixed turbulent flame exhibiting significant levels of local extinction and re-ignition, the Sydney flame L.

The ability of the SOM-MLP approach to provide good regression was assessed at two levels. First, at the development of the tabulation, where new data generated via igniting/extinguishing flamelets were presented to the SOM-MLP and simulated with excellent accuracy. Second, at the application to the turbulent flame, where the LES-PDF-ANN results were compared with those of the LES-PDF-RCCE, and the agreement was also excellent. The ANN tabulation resulted in a reduction of the CPU time was reduced by about 
two orders of magnitude as compared with the real-time RCCE integration and therefore, together with the small storage space required, renders the LES-PDF simulation with comprehensive chemistry feasible on moderate CPU resources such as a workstation.

\section{Acknowledgements}

L. L. C. Franke gratefully acknowledges the support provided by the Brazilian National Council for the Development of Science and Technology $(\mathrm{CNPq})$ through the Science Without Borders program under the Grant 201903/2014-8. The authors also wish to thank Dr Salvador Navarro-Martinez and Prof. W. P. Jones for insightful discussions. This work used the ARCHER UK National Supercomputing Service (http://www.archer.ac.uk).

\section{Bibliography}

[1] S. Lam, D. Goussis, Symp. (Int.) Combust. 22 (1989) 931-941.

[2] U. Maas, S. B. Pope, Combustion and Flame 88 (1992) 239-264.

[3] T. Løvås, Combustion and Flame 156 (2009) 1348-1358.

[4] J. Y. Chen, in: Workshop on Numerical Aspects of Reduction in Chemical Kinetics, CERMICS-ENPC Cite Descartes Champus sur Marne, France.

[5] C. Sung, C. Law, J.-Y. Chen, Symp. (Int.) Combust. 27 (1998) 295-304.

[6] W. P. Jones, S. Rigopoulos, Combustion and Flame 142 (2005) 223-234.

[7] W. P. Jones, S. Rigopoulos, Combustion Theory and Modelling 11 (2007) 755-780.

[8] W. P. Jones, S. Rigopoulos, Proceedings of the Combustion Institute 30 (2005) 1325-1331.

[9] P. Koniavitis, S. Rigopoulos, W. P. Jones, Combustion and Flame 183 (2016) 126-143.

[10] T. Lu, C. K. Law, Progress in Energy and Combustion Science 35 (2009) 192-215.

[11] A. S. Tomlin, T. Turnyi, M. J. Pilling, in: M. Pilling (Ed.), Low-Temperature Combustion and Autoignition, volume Volume 35, Elsevier, 1997, pp. 293-437.

[12] D. A. Goussis, U. Maas, in: Turbulent Combustion Modeling, Springer, 2011, pp. 193-220.

[13] T. Løvås, Model reduction techniques for chemical mechanisms, INTECH Open Access Publisher, 2012.

[14] J. Y. Chen, W. Kollmann, R. W. Dibble, Combustion Science and Technology 64 (1989) 315-346.

[15] S. B. Pope, Combustion Theory and Modelling 1 (1997) 41-63.

[16] J. Kim, S. B. Pope, Combustion Theory and Modelling 18 (2014) 388-413.

[17] F. Christo, A. Masri, E. Nebot, S. B. Pope, Symp. (Int.) Combust. 26 (1996) 43-48.

[18] J. A. Blasco, N. Fueyo, C. Dopazo, J. Ballester, Combustion and Flame 113 (1998) 38-52.

[19] J. A. Blasco, N. Fueyo, C. Dopazo, J. Y. Chen, Combustion Science and Technology 4 (2000) 61-76.

[20] A. K. Chatzopoulos, S. Rigopoulos, Proceedings of the Combustion Institute 34 (2013) 1465-1473.

[21] A. Kempf, F. Flemming, J. Janicka, Proceedings of the Combustion Institute 30 (2005) 557-565.

[22] M. Ihme, C. Schmitt, H. Pitsch, Proceedings of the Combustion Institute 32 (2009) 1527-1535.

[23] B. A. Sen, S. Menon, Proceedings of the Combustion Institute 32 (2009) 1605-1611.

[24] B. A. Sen, S. Menon, Combustion and Flame 157 (2010) 62-74.

[25] A. K. Chatzopoulos, Modelling of Turbulent Combustion, Ph.D. thesis, Imperial College London, London, 2013.

[26] N. Peters, Turbulent Combustion, Cambridge University Press, 2004.

[27] S. Rigopoulos, T. Løvås, Proceedings of the Combustion Institute 32 (2009) 569-576.

[28] T. Løvås, S. Navarro-Martinez, S. Rigopoulos, Proceedings of the Combustion Institute (2011) 1339-1346.

[29] T. Kohonen, Self-Organizing Maps, Springer-Verlag, 2001.

[30] S. Albayarak, Proceedings of the 9th International Conference on Neural Information Procesing 3 (2002).

[31] S. Haykin, Neural Networks: A Comprehensive Foundation, Prentice Hall, 1999. 
[32] C. Bishop, Neural Networks for Pattern Recognition, Clarendon Press, 1995.

[33] Y. Le Cun, Generalization and network design strategies, Technical Report, Department of Computer Science, University of Toronto, 1989.

[34] F. Gao, E. E. O‘Brien, Physics of Fluids (1993) 1282-1284.

[35] R. Mustata, L. Valino, C. Jimenez, W. P. Jones, S. Bondi, Combustion and Flame 145 (2006) 88-104.

[36] W. P. Jones, S. Navarro-Martinez, Combustion and Flame 150 (2007) 170-187.

[37] W. P. Jones, V. N. Prasad, Combustion and Flame 156 (2010) 1621-1636.

[38] J. Smagorinsky, Montlhy Weather Review 91 (1963) 99-164.

[39] J. Villermaux, J. C. Devillon, in: Second International Symposium on Chemical Reaction Engineering, Elsevier, New York, 1972, pp. 1-13.

[40] C. Dopazo, E. O’Brien, Acta Astronautica 1 (1974) 1239-1266.

[41] S. B. Pope, Progress in Energy and Combustion Science 11 (1985) 119-192.

[42] D. Haworth, Progress in Energy and Combustion Science 36 (2010) 168 - 259.

[43] L. Valino, Flow, Turbulence and Combustion 60 (1998) 157-172.

[44] V. Sabelnikov, O. Soulard, Physical Review 72 (2005) 16301-163022.

[45] W. P. Jones, V. N. Prasad, Proceedings of the Combustion Institute 33 (2011) 1355-1363.

[46] W. P. Jones, A. J. Marquis, V. N. Prasad, Combustion and Flame 159 (2012) 3079-3095.

[47] M. Frenklach, H. Wang, M. Goldenberg, G. Smith, D. Golden, C. Bowman, R. Hanson, W. Gardiner, V. Lissianski, GRIMech An Optimized Detailed Chemical Reaction Mechanism for Methane Combustion, Technical Report, Gas Research Institute, 1995.

[48] A. Masri, R. Bilger, R. Dibble, Combustion and Flame (1990) 260-276.

[49] A. R. Masri, W. Dibble, R. S. Barlow, Progress in Energy and Combustion Science (1996) 307-362.

[50] J. Y. Chen, W. Kollmann, R. Dibble, Combustion Science and Technology (1989) 315-346.

[51] A. R. Masri, S. B. Pope, Combustion and Flame 81 (1990) 13-29.

[52] W. P. Jones, M. Kakhi, Combustion and Flame 115 (1998) 210-229.

[53] V. N. Prasad, M. Juddoo, A. R. Masri, W. P. Jones, K. H. Luo, Combustion Theory and Modelling (2013) $483-503$.

[54] H. Wang, M. Juddoo, S. Starner, A. Masri, S. B. Pope, Proceedings of the Combustion Institute (2013) 1251-1259.

[55] S. Elbahloul, S. Rigopoulos, Combust. Flame 162 (2015) 2256-2271. 